\title{
Aplicação da Tecnologia Digital de Informação e Comunicação no Ensino Superior
}

\footnotetext{
Olga Albuquerque ${ }^{1}$, Maria Hosana Conceição', Maria Fernanda Melis $^{1}$, Alberto Mesaque Martins ${ }^{2}$, Felipe Moreira de Albuquerque ${ }^{3}$, Neusi Berbel ${ }^{4}$

1 Programa de Pós-Graduação em Propriedade Intelectual e Transferência de Tecnologia Universidade de Brasília, Brasil | olgamaria@unb.br; hosanac@unb.br; mfernanda.melis@gmail.com | https://orcid.org/0000-0001-6824-603X; https://orcid.org/0000-0002-0823-7841; https://orcid.org/0000-0003-3489-4551

${ }^{2}$ Programa de Pós-Graduação em Psicologia - Universidade Federal do Mato Grosso do Sul, Brasil | alberto.mesaque@ufms.br | https://orcid.org/0000-0002-6032-3122

${ }^{3}$ Escola Politécnica da Universidade de São Paulo, Brasil felipe.moreira.albuquerque@usp.br | https://orcid.org/0000-0002-1998-7939

${ }^{4}$ Professora aposentada da Universidade Estadual de Londrina/PR, Brasil | neusiberbel@gmail.com | https://orcid.org/0000-0000-0000-0000

Resumo: Introdução Este estudo focalizou as Tecnologias Digitais de Informação e Comunicação (TDICs) incorporadas à disciplina Promoção à Saúde 1 (PS1), do curso de Saúde Coletiva de uma universidade pública, na modalidade 'on-line' durante a pandemia de COVID-19; Objetivos; analisar as percepções de estudantes quanto à utilização de TDICs no processo ensino-aprendizagem da PS1; Métodos Pesquisa qualitativa de estudo de caso das respostas dos estudantes ao Microsoft Forms. A análise temática dos dados apontou para os núcleos de sentidos: "avaliação dos recursos digitais para os objetivos de aprendizagem", "consequência da inclusão dos Determinantes Sociais de Saúde (DSS), da atuação intersetorial e da Política Nacional de Promoção da Saúde (PNPS) na formação profissional de Gestor" e "potencial da interação dos grupos para a construção da aprendizagem Resultados $\mathrm{O}$ uso das TDICs foi considerado importante estratégia na mediação dos estudantes com os objetivos/conteúdos trabalhados para sua formação como gestores. Seus depoimentos demonstraram a relevância da inclusão dos DSS, da atuação intersetorial e da formação profissional conectada à PNPS para atuarem como futuros gestores e para desenvolverem habilidades de pensamento crítico. Os relatos desvelam existência de interação nos grupos para atividades de construção conjunta. Conclusões. O uso das TDICs proporcionou experiências múltiplas de aprendizagem, potencializou a apropriação/aplicação dos DSS e das parcerias intersetoriais nas ações registradas no Projeto de Gestor. Assim, o estudo de caso permitiu à professora refletir sobre sua atuação e desenvolver lentes para ampliar a compreensão do modo como os estudantes aprendem a ser/agir no mundo.
}

Palavras-chave: Tecnologia Digital de Informação e Comunicação; Tecnologia Educacional; Recursos Digitais.

Digital Information and Communication Technology Application in Higher Education

Abstract: Introduction: This study focuses on Information and Communication Digital Technologies (ICDT) incorporated to the subject Health Promotion 1 (HP1) of the Public Health undergraduate course in a public university. It has been offered on line since the beginning of the COVID-19 pandemic. Aim: To analyze students' perception of ICDT use in the process of teaching-earning in PS1. Methods: Qualitative research of case study of Microsoft Forms answer sheets filled by the students. The data's thematic analysis disclosed three categories: "digital resources' evaluation for learning objectives", "consequences of including Social Health Determinants (SHD), intersectoral and National Health Promotion Policy (NHPP) action in manager's professional development" and "group interaction's potential to construct learning". Results: ICDT usage is considered an important strategy in the mediation of students with goals and contents prepared to their qualification as managers. Their statements show the relevance of including SHD, intersectoral action to their professional qualification. These issues are linked to NHPP, as subside their act as future managers and to develop critical thinking skills. Students' declarations unveil the existence of interaction in HP1 groups in jointly construct activities. Conclusions: The use of ICDT brought about multiple learning experiences, intensified the appropriation/application of SHD and intersectoral partnerships in actions to promote health as registered in the Manager Project. Hence, the case study made the professor reflect on her function and strive to comprehend how the students learn to be and act in the world.

Keywords: Digital Information and Communication Technology; Educational Technology; Digital Resources. 


\section{Introdução}

As Tecnologias de Informação e Comunicação (TICs) compreendem um conjunto diversificado de ferramentas/recursos tecnológicos usados para comunicar, criar, disseminar, armazenar e gerenciar informações (Blurton, 1999, p. 46). Sua incorporação ao processo de ensino-aprendizagem acompanhou uma tendência observada nas gerações denominadas "nativos digitais" (Prensky, 2001), isto é, sujeitos que nasceram e cresceram em um mundo onde as TICs integram diferentes facetas do cotidiano.

Nas últimas décadas, marcadas por importantes avanços tecnológicos, surgiram as Tecnologias Digitais de Informação e Comunicação (TDIC). Elas promovem a comunicação por meio de sistemas como a internet, que são acessados por diferentes formas (redes sociais, plataformas, aplicativos) e, por meio de vários dispositivos (computadores, tablets, smartphones). Isso inclui a 'mídia social', um modelo de comunicação e interação de muitos para muitos por meio de tecnologias digitais e de 'sites' que possibilitam aos usuários a criação, o compartilhamento ou a troca de conteúdo (Cummings \& O'Neil, 2015). No âmbito da educação, as TDICs consistem em ferramentas e recursos tecnológicos que subsidiam o processo pedagógico mediante a utilização dos softwares educacionais apoiados em uma didática que potencialize o processo de ensinoaprendizagem dos alunos (Budd et al., 2020). Isso ocorre, sobretudo, em situações nas quais o ensino presencial não possa ser praticado, como em contextos de emergências sanitárias.

No início de 2020, a humanidade foi surpreendida pela pandemia da Covid-19 (Coronavirus disease 2019). As medidas de distanciamento social e de controle do contágio exigiram transformações nas práticas pedagógicas como a utilização das TIDCs, inclusive, para formação de profissionais de saúde (Budd et al., 2020). Devido a isso, disciplinas que eram ofertadas em modalidades presenciais e, com forte articulação teórico-prática, foram abruptamente modificadas, exigindo a busca de alternativas. Nesse contexto, situa-se a disciplina de Promoção à Saúde 1 (PS1), do bacharelado em Saúde Coletiva de uma universidade pública brasileira.

A Promoção da Saúde (PS) (Ministério da Saúde, 2002) apresenta diferenças em relação ao corpo de conhecimento que fundamenta a Educação em Saúde (ES). O conceito positivo de saúde, que subjaz à PS, se reflete no desenvolvimento de potencialidades para responder de forma positiva aos desafios do ambiente no cotidiano das pessoas e no atendimento das necessidades apontadas pelos sujeitos. Sua atuação aborda um conjunto de Determinantes Sociais de Saúde (DSS) e preconiza, como estratégia de enfrentamento, o desenvolvimento de parcerias intersetoriais (Batistella, 2007). Nas ações implementadas com a marca da PS, verifica-se um contraste entre a ênfase dada à mudança do estilo de vida das pessoas, predominante na ES, e as estratégias ampliadas que se configuram nos campos de ação da PS: formulação de políticas públicas; construção de ambientes saudáveis; desenvolvimento de habilidades; fortalecimento da ação comunitária e reorientação de serviços (Ministério da Saúde, 2002).

Por essa razão, o aprofundamento dos DSS e das parcerias intersetoriais foram priorizadas como base para atuar em PS. Na oferta da disciplina PS1 'on-line', os estudantes foram instigados a pensar como gestores. Eles foram impelidos a proporem ações sobre os DSS por meio de parcerias intersetoriais, como exercício de planejamento. Nessa perspectiva e, especialmente durante a pandemia de Covid-19, o ensino dos princípios da PS tornou-se mais urgente e desafiador, em vista da necessidade de transição para o ensino não presencial.

O ano letivo de 2020 iniciou de forma presencial. Mas, logo após a constatação dos primeiros casos de Covid-19 foram suspensas as atividades de ensino que, nesta universidade, só foram retomadas em agosto do mesmo ano. Nesse intervalo, o Centro de Ensino à Distância (CEAD) capacitou os professores para utilização das TDICs como ferramentas para a implementação das disciplinas. Além disso o CEAD disponibilizou a versão do Modular Object-Oriented Dynamic Learning (Moodle) como suporte às disciplinas ofertadas 'on-line'. 
O uso do Moodle e das ferramentas tecnológicas que acompanharam esse novo modo de lecionar representava um desafio, pois a disciplina de PS1 era ofertada no formato presencial desde 2009. Seu papel consistia no esforço contínuo para aplicar a teoria ao planejamento, à prática e à execução de ações de PS visando uma formação conectada ao mundo real e às necessidades identificadas pela comunidade em diálogo crítico com os estudantes. O escopo das avaliações era desenvolver a prática reflexiva preconizada por Schön $(1983,1987)$. Para ele o conhecimento profissional se desdobra na racionalidade técnica (regras) e na arte profissional (reflexão na ação). Isso possibilita o resgate da teoria que permeia o fazer profissional podendo melhorar o exercício da profissão, que se configura num processo de pesquisa da teoria na práxis pedagógica, visto que se desenvolve a partir da materialização do exercício docente.

A vivência da reflexão na ação para o aprimoramento da prática profissional na disciplina PS1 (Schön, 1983, 1987) originou a sequência de avaliações efetuadas a cada término de semestre ao longo de 11 anos. Nesse contexto, a pergunta guia deste estudo é: como os estudantes perceberam a inclusão dos recursos digitais na PS1 na modalidade 'on-line?

Esse estudo teve como objetivo analisar as percepções de estudantes de Saúde Coletiva quanto à utilização de TDICs com uso dos recursos digitais e sua influência para o alcance dos objetivos de aprendizagem na disciplina Promoção à Saúde 1.

\section{Metodologia}

Adotou-se o estudo de caso com vistas a "adquirir conhecimento do fenômeno [...] a partir da exploração intensa de um único caso" (Becker, 1993, p. 117). Para Goldenberg (2001, p. 33), esse tipo de pesquisa qualitativa "estuda [...] uma comunidade como um todo, com o objetivo de compreendê-la em seus próprios termos". Trata-se de um estudo de caso intrínseco, visto que a pesquisa é empreendida em razão da singularidade do fenômeno que o pesquisador deseja compreender melhor (Stake, 2000, p. 436).

A comunidade (Goldenberg, 2001, p. 33) está aqui representada pelos 33 estudantes de SC que cursaram a disciplina PS1, com carga horária de 60 horas, ofertada em formato não presencial, no primeiro semestre de 2020. Sua estrutura organizativa se compunha de três unidades para as quais foram utilizadas TDICs e interfaces com ferramentas de aprendizagem. O objetivo metodológico era impulsionar a apropriação dos conceitos próprios da Promoção da Saúde e a aplicação desses saberes em um cenário de simulação, configurado na elaboração do Projeto de Gestor.

Para atingir os objetivos de aprendizagem utilizaram-se as seguintes ferramentas tecnológicas: encontros síncronos dialógicos e gravações para permitirem o acesso assíncrono na plataforma Moodle; uso de atividades interativas mediadas por tecnologias digitais como vídeos do YouTube; produção criativa de 'podcast' e de um mapa mental como atividade colaborativa dos grupos; leitura, análise de artigos científicos sobre a PS e, subsequente inserção dos conceitos no Projeto de Gestor; criação de um grupo de WhatsApp para comunicação com os monitores; e, a avaliação final da disciplina por meio do Microsoft Forms.

Inicialmente promoveu-se a ambientação dos estudantes na plataforma Moodle criando as condições para interagirem com diferentes recursos digitais: a apresentação no Fórum para compartilhar sua disposição em reiniciar as atividades nessa nova modalidade não presencial; a atualização de seu perfil com uma foto que ajudasse em sua identificação; a inclusão de palavras no Mentimeter para construção de uma nuvem de palavras que subsidiaram a discussão sobre os DSS partindo do entendimento prévio dos estudantes acerca desse tema; a linha do tempo elaborada com o HiperText Markup Language 5 Package (H5P), que evidenciava a incorporação do ambiente e da participação da comunidade ao processo saúde doença. 
A cada encontro síncrono efetuavam-se avaliações da disciplina PS1 para aprimorar sua organização à medida que os estudantes utilizavam o Moodle. Os comentários deles eram anotados para subsidiar as correções das dificuldades apontadas ao navegarem na plataforma no decorrer do semestre letivo. Suas contribuições incluíram: a antecipação para o início do semestre da liberação de todas as atividades na abertura do tópico e do esquema básico do Projeto de Gestor, além da inserção de aspectos da Política Nacional de Promoção da Saúde (PNPS) (Ministério da Saúde, 2018) ao passo que os conceitos da PS fossem apresentados. Esse processo de construção coletiva da PS1 ativou a apropriação gradual dos conceitos próprios da PS e a associação à PNPS (Ministério da Saúde, 2018).

Para a avaliação final da disciplina aplicou-se um questionário via Microsoft Forms, que ficou disponível aos estudantes por sete dias na antepenúltima semana antes do término do semestre com as seguintes perguntas: 1. Como você avalia os recursos digitais utilizados em relação aos objetivos de aprendizagem da PS1? 2. O que você pensa da inclusão dos DSS e a subsequente atuação intersetorial para sua formação profissional de Gestor de PS? 3. O que você tem a dizer sobre a utilização da PNPS para seu futuro exercício profissional como Gestor? 4. Ao longo do semestre você interagiu com seus colegas nos grupos para a realização das atividades e para a construção da aprendizagem? Se você respondeu sim, escreva aqui as consequências dessa interação.

A análise de conteúdo das respostas dos estudantes ao Microsoft Forms empregou o tema como regra de recorte. O "tema é [...] utilizado como registro para estudar motivações de opiniões, de valores, de crenças, de tendências etc". Segundo Bardin (2011, p. 135) é adequada a aplicação do tema na "análise das respostas a perguntas abertas" como se fez nesse estudo. A unidade de registro "corresponde ao segmento de conteúdo considerado unidade de base visando a categorização" e pode se configurar em "uma palavra ou em uma frase" (Bardin, 2011, p. 134).

Inicialmente procedeu-se à leitura flutuante para identificar os principais temas que emergiram das respostas anônimas dos estudantes ao questionário. O percurso desta etapa desvelou informações contidas nas frases-reposta. Na sequência, a exploração do material propiciou a captura das expressões heurísticas com base empírica, matrizes da reflexão sobre a reflexão na ação acerca da prática pedagógica adotada na PS1. A análise temática dos dados apontou para núcleos de sentido relacionados à "avaliação dos recursos digitais para os objetivos de aprendizagem"; à "consequência da inclusão dos DSS/atuação intersetorial e da PNPS na formação profissional de Gestor de PS" e ao "potencial da interação dos grupos para a construção da aprendizagem".

O processo de categorização efetuado incialmente por NB e revisitado por OA ressaltou aspectos pensados desde a elaboração do instrumento de coleta dos dados. Tal instrumento fornecia o sistema de categorias pelo procedimento de "caixas" (Bardin, 2011, p. 149). A organização do material, a inferência e a interpretação dos dados analisados resultou do diálogo efetuado entre a base teórica que orientou as leituras, a construção da pergunta e o objetivo deste estudo. Nos resultados os estudantes estão identificados com a letra "E" seguida pelo número que indica a sequência de respostas ao Microsoft Forms.

\section{Resultados}

As informações qualitativas geradas pelas respostas dos estudantes compuseram o 'corpus' que apontou para três categorias principais, organizadas nos eixos de percepção: "avaliação dos recursos digitais para os objetivos de aprendizagem"; "inclusão dos DSS/atuação intersetorial e da PNPS (Ministério da Saúde, 2018) na formação profissional de Gestor de PS" e "potencial da interação dos grupos para a construção da aprendizagem". 


\subsection{Avaliação dos Recursos Digitais para os Objetivos de Aprendizagem}

A passagem do formato presencial para 'on-line' foi motivo de adaptação para professores e estudantes, com aparente sobrecarga de trabalhos e de solicitação de tarefas. Isso resultou em uma taxa de $67 \%$ de resposta ao questionário. O repertório de enunciados dos respondentes demonstrou o diferencial que as ferramentas tecnológicas e de rede representaram no cenário da educação: "Achei muito didático [...] ajudou muito na compreensão dos temas" (E6); "Foram essenciais na assimilação do conteúdo [...] torna a disciplina mais interativa. Tem pessoas que aprendem de maneira mais visual, outras gostam de ler, alguns de ouvir áudios, enfim quanto maior a diversidade de recursos utilizados, maior é a possibilidade de que os alunos assimilem melhor o conteúdo" (E7).

Um argumento amadurecido, do ponto de vista organizativo, se destacou em meio ao conjunto de fragmentos expressivos de concordância com a inclusão dos recursos digitais na PS1: "Achei ótimo usar diferentes recursos para o melhor aprendizado da matéria, mas acho que o podcast para o trabalho do Gestor poderia ter ficado para o final do semestre" (E16). Sua sugestão foi atendida pela professora ao elaborar a sequência de atividades do semestre subsequente. A reflexão sobre a ação ancorada na avaliação dos estudantes, fomentou o processo de aprendizagem contínua, que é uma faceta determinante para o praticante reflexivo (Schön, 1987).

A motivação da proposta de $\mathrm{E} 16$ se relaciona ao fato que todos os estudantes têm muita clareza ao enumerar os DSS. Entretanto, ao fazerem o exercício intelectual de sua aplicação nas tarefas propostas, isso parece se situar numa esfera que extrapola sua área de atuação. Esse tipo de divergência entre o discurso e a aplicação prática dos conceitos reflete a predominância do modelo biomédico/comportamental que persiste, apesar de passados 35 anos da inclusão dos DSS a uma concepção de saúde mais abrangente e contemporânea, como base para atuação intersetorial e de promoção da saúde (Batistella, 2007).

As mudanças ocorridas no conceito de saúde e reportadas na linha do tempo foram associadas ao conceito positivo de saúde da Carta de Ottawa (Ministério da Saúde, 2002, p. 19) e à incorporação dos DSS à legislação brasileira que organiza o Sistema de Saúde brasileiro. Para evidenciar o entendimento dessas mudanças solicitava-se a "inserção de propostas com iniciativa de incorporação dos DSS e do conceito ampliado de saúde no Projeto de Gestor".

A avaliação de E18 deixa transparecer algumas dificuldades: "no começo do semestre foi um pouco confuso alguns pontos que estavam nos Tópicos, mas a partir do Tópico 3, ficaram claros". Estas dificuldades persistiram até os estudantes se apropriarem, progressivamente, das leituras disponíveis nos Tópicos subsequentes, como relata E10: "No início senti algumas dificuldades para compreender o que era para ser feito, mas depois consegui entender melhor o que a professora estava pedindo no decorrer dos encontros, pois o conteúdo ajudou a entender bastante. E por fim, mesmo com essa dificuldade, essa foi uma das poucas disciplinas que cursei esse semestre e posso dizer que absorvi o conteúdo dado e aprendi".

Passado o momento de dúvidas e de esclarecimentos, os recursos digitais foram reconhecidos como importantes por $78 \%$ dos estudantes na mediação com os objetivos e os conteúdos trabalhados numa situação real de estudo com vistas à sua formação como gestores. Importante ressaltar a dinâmica de interação constante entre a professora e os estudantes para dar um sentido comum à proposta pedagógica e reconstruírem juntos detalhes para o formato mais adequado ao grupo. O papel de praticante reflexivo no exercício de docência resultou da reflexão na ação efetuada com os estudantes da disciplina PS1. 


\subsection{Consequência da Inclusão dos DSS/Atuação Intersetorial e da PNPS na Formação Profissional de Gestor de PS}

Observou-se consenso entre os estudantes acerca da inclusão dos DSS e da PNPS (Ministério da Saúde, 2018) na PS1. Isso se revela nas respostas que conectam estes conteúdos à própria formação como futuros profissionais: "A inclusão dos DSS e a atuação intersetorial na minha formação de gestora é muito importante para nosso aprendizado porque tudo implicará no meu futuro [...] para saber administrar a minha profissão e ajudar a trabalhar juntamente com meus colegas para o melhoramento da saúde pública do país" (E8). Sua percepção é compartilhada por E12 ao afirmar que "para a atuação profissional como gestor é importante ter o conhecimento desses dois temas [DSS e parcerias [intersetoriais] [...] e o conhecimento da PNPS [...] na hora de tomar decisões".

A relevância da aplicação dos DSS e da PNPS (Ministério da Saúde, 2018) na atuação em saúde, perscrutando o território, para além da Unidade de Saúde e conectado às necessidades da sua potencial clientela foi reconhecida por E22: "[...] me ajudou a enxergar a saúde para fora do hospital, para o dia a dia do indivíduo em casa, no trabalho, na escola [...] a PNPS me ajuda a atuar utilizando políticas públicas já existentes".

Este conjunto de expressões dos estudantes reflete um raciocínio heurístico, que substitui a habitual oposição entre "saber e fazer" e materializa a vinculação simbiótica entre estes. A construção coletiva da aprendizagem para estimular a inserção dos conceitos e da base legal no Projeto de Gestor gerou o exercício de aplicação dos conteúdos pelos estudantes antevendo sua atuação como profissionais. Isso permitiu deslocar o "eixo do trabalho pedagógico da compreensão intelectual para a atividade prática, do aspecto lógico para o psicológico, dos conteúdos cognitivos para os métodos ou processos de aprendizagem" (Saviani, 2007).

\subsection{Potencial da Interação dos Grupos para a Construção da Aprendizagem}

A realização dos trabalhos em grupo é considerada importante como formadora para profissionais de saúde, promovendo aprendizagem colaborativa (Hassanien, 2006) e melhorando seu desempenho e socialização (Frykedal \& Chiriac, 2011). De igual modo, a escuta é importante para o desenvolvimento de trabalhos conjuntos e o acolhimento dos usuários que procuram os serviços de saúde. O ganho nas interações foi relatado por $87 \%$ dos respondentes relacionados à troca de conhecimentos/ideias/opiniões (E3) e apoio no uso da plataforma (E12). A avaliação de E18 aponta para: a "troca de saberes entre os colegas com diferentes opiniões, mais empatia para entender os horários e união entre os integrantes". E E8 destaca: "Realizamos todos juntos o 'podcast'. Tudo foi bem administrado com meus colegas de turma e soubemos fazer o trabalho".

Entretanto, essa visão não é homogênea. Outros estudantes manifestaram dificuldade de interação nos grupos de trabalho na PS1 e salientaram um desafio que ainda precisa ser superado em trabalhos de grupo. E6 afirmou: "a interação com os colegas de grupo foi bem difícil durante todo o semestre, [...] via mensagens [...], sendo assim, a realização de atividades em grupo ficou prejudicada"; E22 acrescentou "Não foram consequências muito positivas [...] sempre têm os que fazem os trabalhos e os que são levados pelos outros". A menção à experiência estressante de E6 e E22 foi descrita por Hall \& Buzwell (2012), especialmente para aqueles membros ativos do grupo.

O olhar positivo de E10 sobre sua experiência na PS1 contrasta com vivências anteriores: "Não gosto muito da ideia de trabalhos em grupo, pois muitas vezes não temos a participação efetiva de todos os membros [...]. Mas, essa disciplina me proporcionou algo que nunca tinha vivenciado: processo de colaboração de todos os integrantes do grupo e acima de tudo todos tinham um espaço de fala e para ser ouvido no grupo". A dialogicidade na disciplina propiciada pela professora foi reconhecida por E8: "[...] gostei bastante porque ela [a professora] sempre ouviu todas as nossas opiniões." 
Segundo Mishra e Mehta (2017, p. 16) "para que uma disciplina estimule e envolva as pessoas, ela precisa abrir espaço para o pensamento criativo, crítico e apoiar as ideias, a colaboração e a comunicação inovadoras".

Dentre os 23 respondentes 20 desvelaram a existência de interação ao desenvolverem as atividades colaborativas nos grupos da PS1: "troca de conhecimentos, organização na realização dos trabalhos, divisão de tarefas e diálogos relacionados a melhor realização das tarefas" (E20). Eles avaliaram o vínculo construído no grupo como "muito bom, pois sem a ajuda dos meus colegas [eu] não teria conseguido realizar algumas atividades e participar de alguns encontros devido às dificuldades com a plataforma" (E23).

\section{Conclusões}

Este estudo apresenta o desenvolvimento da disciplina PS1 como corpo/cenário do caso estudado. Efetuaram-se avaliações formativas quanto ao seu andamento ao longo do semestre em cada encontro síncrono, por ser a primeira oferta no formato não presencial utilizando as TDICs. Sob este prisma o 'caso' aqui descrito não se distingue do contexto de vida real com seus desafios de comunicação para o desenvolvimento de uma prática reflexiva junto com os estudantes.

Desse modo, o estudo de caso permitiu à professora refletir sobre sua atuação, ultrapassar os fatos e desenvolver lentes para ampliar a compreensão do modo como os estudantes aprendem a ser e a agir no mundo. O método mostrou-se apropriado para retratar e apreender a repercussão do emprego das TDICs na ampliação da capacidade cognitiva dos estudantes em cenário virtual; adequado para capturar novas conexões estabelecidas entre os saberes acumulados e a futura atuação profissional, bem como para acrescentar peculiaridades à construção e à troca de saberes entre os estudantes e destes com a professora.

É possível afirmar que a utilização das TDICs forneceu a base estrutural para os estudantes vivenciarem experiências múltiplas de aprendizagem. Isso potencializou a apropriação/aplicação dos DSS e das parcerias intersetoriais nas ações para promover saúde ao construírem coletivamente o Projeto de Gestor. Na percepção dos estudantes, estes elementos foram relevantes para sua formação profissional e para a interação gerada nos grupos, melhorando seu desempenho na realização das atividades e no desenvolvimento das habilidades de pensamento crítico.

O exercício de escuta e de reflexão na ação possibilitaram avaliações e a correção dos rumos na PS1 frente aos objetivos de aprendizagem. Ressalta-se a contribuição substancial dos estudantes para os ajustes efetuados ao longo do semestre em foco e para a organização dos semestres seguintes incorporando as TDICs. As avaliações da PS1 confirmaram a importância de manter aberto o canal de comunicação com eles para o atendimento de suas solicitações e para a construção conjunta da disciplina. Ainda que estudantes e professora desempenhassem papéis distintos, o exercício da reflexão crítica no ensino-aprendizagem suscitou um clima igualitário e participativo.

Desse modo, a compreensão do contexto e da dinâmica da PS1, da abordagem pedagógica utilizada e das possibilidades ampliadas pelas ferramentas tecnológicas, tornam o ensino-aprendizagem uma atividade rica e envolvente. Estudos de caso específicos realizados reiteradamente a cada turma diferente, podem contribuir para a recomposição e o aperfeiçoamento do objeto de estudo, além de constituir uma referência na atuação do praticante reflexivo. 


\section{Referências}

Bardin, L. (2011). Análise de conteúdo. (3. Reimp.). Lisboa: Edições 70.

Batistella, C. (2007). Abordagens contemporâneas do conteúdo do conceito de saúde. In A. F. Fonseca \& A. M. D. Corbo (Orgs.) O território e o processo saúde-doença. (Coleção Educação Profissional e Docência em Saúde: a Formação e o Trabalho do Agente Comunitário de Saúde, 1, pp. 51-86). Rio de Janeiro: EPSJV; FIOCRUZ.

Becker, H. S. (1993). Métodos de pesquisa em ciências sociais. São Paulo: Hucitec.

Blurton, C. (1999). New directions in education: challenges in education. In G. Bartagnon. \& Y. Courrier (Eds.), World communication an information report 1999-2000 (pp. 46-61). Paris: UNESCO.

Budd, J., Miller, B.S., Manning, E.M., Lmapos, V., Zhuang, M., Edelsteins, M., Rees, G. Emerey, V. C., Stevens, M. M., Keegan, N., Short, M. J., Pillay, D., Manley, E., Cox, I. J., Heymann, D., Johnson, A. M., McKendry, R. A. (2020). Digital technologies in the public-health response to COVID-19. Nature Med, 26, 1183-1192.

Cummings, C. \& O'Neil, T. (2015). Do digital information and communications technologies increase the voice and influence of women and girls?: A rapid review of the evidence. Shaping policy for development (March, 2015). London: ODI Reports.

Frykedal, K. F., \& Chiriac, E. H. (2011). Assessment of students' learning when working in groups. Educational Research, 53(3), 331-345.

Goldenberg, M. (2001). A arte de pesquisar: como fazer pesquisa qualitativa em Ciências Sociais. (5. Ed.). Rio de Janeiro: Record.

Hall, D. \& Buzwell, S. (2012). The problem of free riding in group projects: looking beyond social loafing as reason for non-contribution. Active Learning in Higher Education, 14(1), 37-49.

Hassanien, A. (2006). Student experience of group work and group assessment in higher education. Journal of Teaching in Travel \& Tourism, 6(1), 17-39.

Ministério da Saúde (2002). As cartas da promoção da saúde (Série B. Textos Básicos em Saúde). Brasília: Ministério da Saúde.

Ministério da Saúde (2018). Política Nacional de Promoção da Saúde: Anexo I da Portaria de Consolidação no 2, de 28 de setembro de 2017. Brasília: Ministério da Saúde.

Mishra, P. \& Mehta, R. (2017). What we educators get wrong about 21st- century learning: results of a survey. Journal of Digital Learning in Teacher Education, 33(1), 6-19.

Prensky, M. (2001). Digital natives, digital immigrants' part 1. On the Horizon, 9(5), 1-6.

Saviani, D. (2007). Pedagogia: o espaço da educação na universidade. Cadernos de Pesquisa, 37(130), 9-134.

Schön, D. (1983) The Reflective Practitioner. London: Temple Smith.

Schön, D. (1987) Educating the Reflective Practitioner. San Francisco, CA: Jossey-Bass.

Stake, R. (2000). Case studies. In N. K. Denzin, Y. S. Lincoln. Handbook of qualitative research. (2nd ed.). California: Sage. 